\title{
Reduction of Corona Discharge Using TiO2 Coating Treatment for High Voltage Transmission Line
}

\author{
L J. Feng ${ }^{1,2^{\star}}$, H N.Ma $^{1}$, W. N. Shen ${ }^{1}$, G. C. Wang, A L. Lei ${ }^{1}$, B. Deng ${ }^{1}$, H T. \\ $\mathrm{Wei}^{1}$ \\ ${ }^{1}$ Xi'an University of Technology, Xi'an 710048, China \\ ${ }^{2}$ Key Lab. of Corrosion and Protection of Shannxi Province, Xi'an 710048, China \\ *fenglajun@email.com
}

Keywords: corona discharge, transmission line, $\mathrm{TiO}_{2}$ coating, electric field intensity, wetting property

\begin{abstract}
TiO}_{2}$ ceramic coating was prepared by plasma spray process. The roughness and contact angle of coated surface were measured. The droplet morphology was analyzed and the corona discharge occurred on the transmission conductor surface under high voltage (HV) electric field was observed by ultraviolet camera. The results showed that by means of spraying $\mathrm{TiO}_{2}$ coating onto the conductor surface, the reduce in resultant of gravity and electric field force exerting downwards the droplet affixed to the conductor surface led to larger radius of curvature at the tips of water drops. A coarse coating was realized because the surface roughness of the conductor increased from $0.12 \mu \mathrm{m}$ to $5.89 \mu \mathrm{m}$ while the contact angle decreased from $52^{\circ}$ to $21.5^{\circ}$. The conductor without coating showed sparks at $50 \mathrm{kV}$ while merely sparked occasionally at $80 \mathrm{kV}$ for with coating. The wet conductor with coating made considerably less and weaker sparks than the dry conductor without coating.
\end{abstract}

\section{Introduction}

With the increasing demands in scale and capacity of power system, a widespread application of higher voltage class of transmission lines and substations is emerged, however, the application of high voltage is supposed to significantly exacerbate the inhomogeneity of electric field existing in the ambient air, as a result, corona discharge is aroused to produce noise and electromagnetic interference, for these not only electric energy is lost greatly, but also the life of residents located along transmission lines is disturbed seriously, even worse, electromagnetic radiation accompanied in the process is harmful to the health of residents [1]. One of the cognitions to reasons for corona discharge is the unsmooth surface of transmission line with adherence of foreign materials, such as dust, which become a trigger of point effect to produce corona discharge, as well as under another serious condition of rainy day, the distortion of electric field around the top of water drop brings the air nearby into ionization process, so that corona discharge occurs [2]. It has been reported that for transmission lines if the wettability of rain drops on the surface performed higher, its hydrophilic behavior would grow larger, therefore, it is possible for rain drops to make less distorted electric field and softer noise. If extremely hydrophilic behavior is achieved, corona noise would be reduced significantly [3]. Due to advantages such as good stability, without decomposition, excellent dielectric constant, and be capable of degrading dust and decreasing corona discharge on the transmission line surface, in this work, $\mathrm{TiO}_{2}$ coating prepared by plasma spray [4-6] was adopted as surface layer for transmission line to reduce the surface conductance and sectional leakage current of lines, as well as to increase the potential difference between internal and external side of conductor consequently to decrease voltage class of transmission lines. Water drops on the $\mathrm{TiO}_{2}$ ceramic coating tend to grow together to stretch into a continuous film rather than discontinuous and independent drops, because the excellent hydrophilicity is available for $\mathrm{TiO}_{2}$ ceramic to reduce corona origin. Furthermore, $\mathrm{TiO}_{2}$ with possession of excellent photocatalytic properties [7] makes direct contributions to catalytic decomposition of dust on the transmission line surface, so as to 
decrease corona triggered by the attached dust, this, of course, can decrease corona discharge to a large extent.

\section{Experimental procedure}

$\mathrm{TiO}_{2}$ powder fabricated by Beijing General Research Institution of Mining \& Metallurgy with particle size $40 \pm 20 \mu \mathrm{m}$ was used in this study. $\mathrm{TiO}_{2}$ ceramic coating was obtained by plasma spray carried out on a GP80 spray system. Aluminum cable steel reinforced (ACSR) with length $20 \mathrm{~cm}$ and sectional area $38 \mathrm{~mm}^{2}$, composed of 24 aluminum wires surrounding 7 steel wires, was selected as sample for high voltage conductor. High voltage strand conductor used as metal substrate in this work was firstly sand blast treated to remove oxide layer for surface activation [8], and then was cleaned for avoiding any oil contamination on the surface with acetone. Finally, the cleaned surface was immediately plasma sprayed to prevent surface oxidation. The spraying conditions are given in Table 1, according to this spraying arrangement, $\mathrm{TiO}_{2}$ powder was instantly heated with GP80 plasma spraying system for atomization and then deposited on metal substrate to form a structure of ceramic coating.

Table 1 Parameter settings for plasma spraying in this work

\begin{tabular}{cc}
\hline Parameter & Setting \\
\hline Hydrogen pressure & $0.32 \mathrm{Mpa}$ \\
Argon pressure & $0.85 \mathrm{Mpa}$ \\
Voltage & $60 \mathrm{~V}$ \\
Current & $600 \mathrm{~A}$ \\
Spraying distance ${ }^{\mathrm{a}}$ & $150 \mathrm{~mm}$ \\
Deposition rate & $5 \mathrm{Kg} / \mathrm{h}$ \\
Coating thickness & $0.2-0.3 \mathrm{~mm}$ \\
\hline
\end{tabular}

${ }^{a}$ Increasing spraying distance to reduce impact of plasma arc on coating

A contact angle measurement apparatus (JC2000CD) was used to test static contact angles of the conductors with and without $\mathrm{TiO}_{2}$ coating. The state change of water drops on the surface of conductors in rainy day was observed and photographic recorded for following discussion. SURFCORDER.SE3500 surface roughness tool was used to obtain surface roughness before and after $\mathrm{TiO}_{2}$ spraying treatment. The conductors with and without $\mathrm{TiO}_{2}$ coating were placed and arranged in a special test station for high voltage test. In order to simulate corona discharge in rainy day, the conductor surface was sprayed with water before applying a high voltage, $50-110 \mathrm{kV}$ at 50 $\mathrm{Hz}$ of frequency, then the corona discharge condition was observed by ultraviolet (UV) camera at every increase of $10 \mathrm{kV}$ with duration time of $10 \mathrm{~min}$.

\section{Results and discussion}

\subsection{Distribution state of water drops on conductor surface and coating contact angle}

Figure 1 shows distribution of water drops on the surface of conductor in performing simulation of rainy day, and Figure 2 shows measurement result of contact angle on the surface of conductor. It can be seen from Fig. 1 that distribution of water drops on the surface of black conductor with spraying $\mathrm{TiO}_{2}$ undergoes dramatic difference with the silver conductor without spraying treatment. For example, there is a large amount of water drops which naturally hangs on the silver conductor all over the surface, as if the rain is being continued, these small water drops would grow into larger size and gather together on the lowest surface of the conductor, however, water drops are barely absorbed onto the surface of black conductor and merely two or three drops hang on the lowest surface, indicating a good wetting property of $\mathrm{TiO}_{2}$ ceramic coating. It is clear that the wetting property for black conductor with $\mathrm{TiO}_{2}$ coating is greatly improved by comparison with that without coating. Also, this is easy to be proved by measurement of contact angles on conductor surface as 
shown in Fig. 2. It can be observed from Fig. 2 that contact angle $21.5^{\circ}$ is obtained from conductor with $\mathrm{TiO}_{2}$ coating, while $52^{\circ}$ for without coating. Therefore, through investigation of water drops distribution and contact angle of the conductor with and without coating, it is easy to demonstrate that spraying $\mathrm{TiO}_{2}$ is a feasible way to improve surface wettability of the conductor, because the amount of water drops absorbed on the conductor surface and the contact angle are greatly reduced, as a result, the corona discharge triggered by water drops would be reduced accordingly.

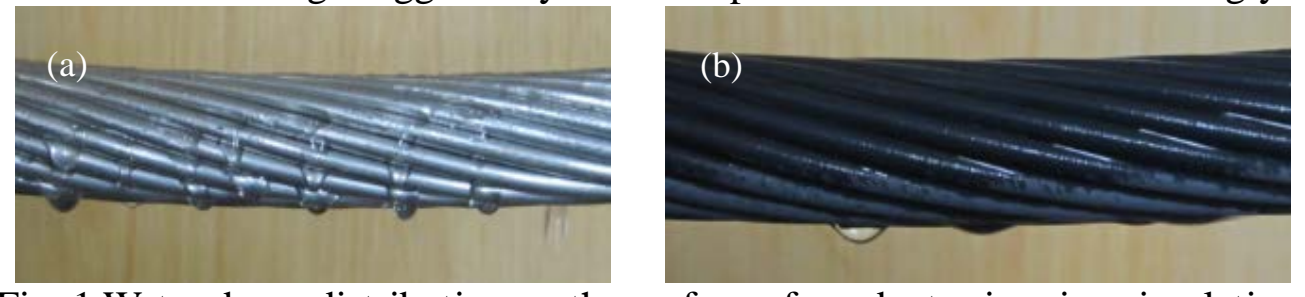

Fig. 1 Water drops distribution on the surface of conductor in rainy simulation,

(a) Before spraying; (b) After spraying with $\mathrm{TiO}_{2}$

(a)

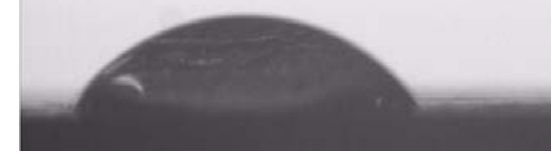

(b)

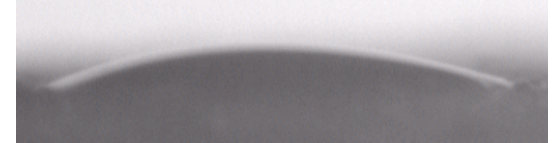

Fig. 2 Contact angle on the surface of conductor

(a) $52^{\circ}$ before spraying; (b) $21.5^{\circ}$ after spraying with $\mathrm{TiO}_{2}$

\subsection{The influence of relative dielectric constant of TiO2 coating on corona discharge}

When it rains, water drops affixed to the lower surface of conductor are polarized under the effect of applied electric field, along with their own gravities these drops are elongated along the direction of gravitational field and almost into an axiolitic shape with small radius of curvature. The connection between radius of curvature and surface electric field intensity of uniform charged sphere is indicated as follows:

$$
\frac{E_{r_{1}}}{E_{r_{2}}}=\frac{r_{2}}{r_{1}}
$$

Based on the Equation (1), it can be found that the radius of curvature at the tip of water drop is smaller than the radius of conductor, therefore, the electric field intensity existing in the interface between air and the tip of water drop shall be much greater than that on the surface of conductor [9].

In rainy or foggy condition, water drops attached to the transmission conductor under operation are elongated gradually by the action of gravity and electric field force, the longer the water drop is elongated, the smaller the radius of curvature at drop tip becomes, as a result, electric field around the conductor surface is distorted greater. The distribution of electric field around conductor is changed by attached water drops, in return the electric field force act on the droplet and stretch it to form a sharp point resulting in distortion of the electric field at the droplet tip where the field intensity is suddenly increased to nearly 3 times as high as that of this place when rain or fog is not available. Based on this distortion of electric field, the threshold of corona voltage is largely decreased namely it is possible for the droplet to trigger the corona with relatively lower applied voltage [10].

If there is the presence of polarization for dielectric in external electric field, the polarized charges would respond with an electric field inside the dielectric which existed as feedback electric field that always plays a role in weakening the polarization, and in most cases it is called depolarization field.

According to superposition principle of electric field intensity, field intensity $\mathrm{E}$ at any point should be equal to vector sum of applied electric field intensity $\left(E_{0}\right)$ and feedback electric field(E produced by polarized charges being there.

$$
\vec{E}=\vec{E}_{0}+\vec{E}^{\prime}
$$


When electric field is uniformly filled with dielectric or for which the equipotential surface is described as shown in Figure 3, the electric field E inside the dielectric could be described as below:

$$
E=\frac{E_{0}}{\varepsilon_{r}}
$$

Here, $\varepsilon_{\mathrm{r}}$ is the relative dielectric constant.

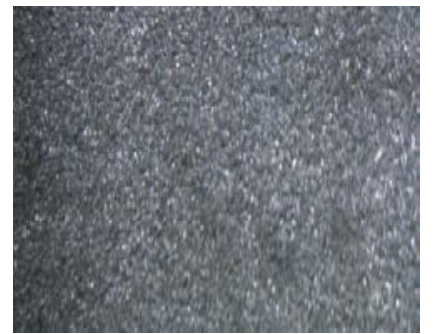

Fig. 3 Equipotential surface

Considering $\mathrm{TiO}_{2}$ coating used as dielectric was polarized by electric field, it appears that the bound charges (also known as polarization charges) were formed both within the coating and on the surface. Since the polarization charges have a considerably less amount than the induced charges emerging on the conductor surface, the electric field within the coating is bound to be weakened under the superposition of feedback electric field $E_{1}$ with applied electric field $E_{0}[11]$.

$$
\vec{E}=\vec{E}_{0}+\vec{E}_{1}<\vec{E}_{0}
$$

Connecting Equation (3) with Equation (4), below equation is then obtained:

$$
E=E_{0}-E_{1}=E_{0} / \varepsilon_{r}
$$

The relative dielectric constant of $\mathrm{TiO}_{2}$ is 100 while the relative dielectric constant of air is 1.0005. The $\mathrm{TiO}_{2}$ coating became an equipotential surface, of which the electric field is 100 times lessened by comparison with the original conductor without coating if it is assumed that the charges on the inner surface of coating is placed under a condition of consistence. Therefore, the electric field force is significantly decreased, bringing about the decrease of resultant force exerting downwards the droplet to a large extent. When the stretching force on the droplet is decreased, it is discovered that the distortion degree of electric field on the conductor surface is weakened, which would result in less strength of corona discharge accordingly.

\subsection{Effect of coating surface roughness on corona discharge}

In carrying out plasma spray with $\mathrm{TiO}_{2}$, a high-temperature arc was formed and heated $\mathrm{TiO}_{2}$ powder to molten or partially molten state, then the plasma was directed to blow the molten powder onto conductor surface to form coating. When molten or partially molten powders sticking to the conductor were cured, the bonding with conductor was constructed, finally, a continuous spraying system enabled particles to stack together to form coating onto the bonding. Thus, it seems clear that depending on the spraying technology, it is inevitable to produce a coarse coating involved in molten and partially molten particles.

The surface roughness of conductor with and without $\mathrm{TiO}_{2}$ coating was analyzed by roughmeter SURFCORDER.SE3500, the results are revealed in Figure 4. It can be observed by comparison Fig.4 (a) with Fig.4 (b), Ra of original conductor is measured as $0.12 \mu \mathrm{m}$ while Ra $5.89 \mu \mathrm{m}$ for with $\mathrm{TiO}_{2}$ coating. As for ideal surface tension, it is defined as below [12]:

$$
\gamma=\left(\frac{\partial G}{\partial A}\right)_{T, P}
$$

By sandblasting treatment, however, the actual surface of the conductor become apparently larger than the surface getting from macro measurement, bringing about the change of surface free energy (described in Equation(6)) as shown below[12]:

$$
d G=\left(\frac{\partial G}{\partial A_{S V}}\right)\left(\frac{\partial A_{S V}}{\partial a_{S V}}\right) d a_{S V}+\left(\frac{\partial G}{\partial A_{S L}}\right)\left(\frac{\partial A}{\partial a_{S L}}\right) d a_{S L}+\left(\frac{\partial G}{\partial A_{L V}}\right)\left(-\frac{\partial A_{L V}}{\partial a_{L V}}\right) d a_{L V}=0
$$



area).

Here, "A" is the actual area of conductor, and "a" denotes the apparent area (namely geometric Further we have Eq.(7) divided by dasv, since da $\mathrm{sv}_{\mathrm{SV}}=-\mathrm{da}_{\mathrm{SL}}$, then $\frac{d a_{L V}}{d a_{S L}}=\cos \theta^{\prime}$ And set $\beta=\frac{A}{a}=\frac{d A}{d a}, \beta$ is defined as roughness actor.

Then the following Equation is obtained:

$\beta\left(\gamma_{S V}-\gamma_{L V}\right)=\gamma_{L V} \cos \theta$

Consider ideal surface, we have $\cos \theta=\frac{\gamma_{S V}-\gamma_{S L}}{\gamma_{L V}}$ (9). Substituting Equation (9) into Equation

(8), $\beta$ is obtained $\beta=\frac{\cos \theta^{\prime}}{\cos \theta}(10)$

(a)

$$
\text { P.profile } \longmapsto 0.2 \mathrm{~mm}
$$

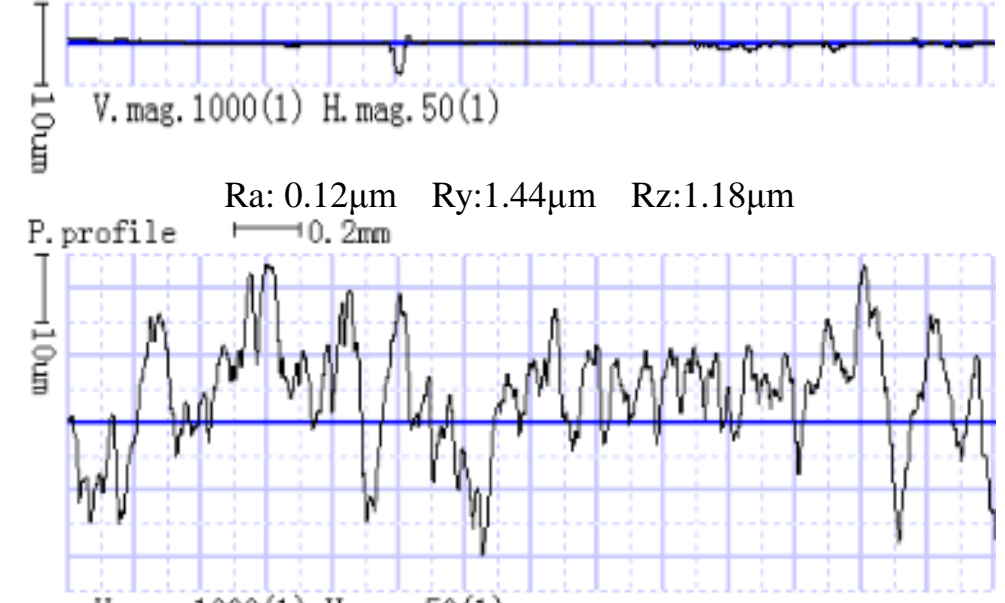

V. mag. 1000 (1) H. mag. 50(1)

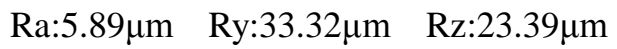

Fig.4 Surface roughness analysis of conductor with and without $\mathrm{TiO}_{2}$ coating,

(a) Original conductor without $\mathrm{TiO}_{2}$ coating and (b) conductor with $\mathrm{TiO}_{2}$ coating

As for coarse surface, it has $\beta>1$, and substituting it into Equation (10) to get $\theta^{\prime}<\theta$, this indicates that the wetting property of a coarse surface is superior to a smooth surface. It has ever been reported that during rainy or foggy day, water drops attached to the transmission conductor under operation was elongated gradually by gravity and electric field force, the longer that water drop was elongated, the smaller the radius of curvature at the drop tip became, as a result, electric field around the conductor surface was distorted greater [13]. Therefore, if the wetting property of conductor surface is increased to a certain extent, when a water drop is dropped onto this surface, it is easier to be stretched into a broad film rather than a spherical droplet, so that tips of conical water drops disappear a lot. It can be concluded from the above steps that making a surface rougher to increase its wetting property is a favorable way to reduce the corona discharge.

\subsection{Corona discharge testing}

Figure 5 shows the image of corona discharge on conductor surface. The corona discharge of conductor without $\mathrm{TiO}_{2}$ coating under $110 \mathrm{kV}$ condition are shown in Fig. 5(a) and Fig. 5(b), of which Fig. 5(a) indicates the discharge spark for dry conductor while Fig. 5(b) for wet conductor. As for conductor with $\mathrm{TiO}_{2}$ coating, Fig. 5(c) and Fig. 5(d) correspond to spark of dry and wet conductor, respectively. It is revealed by comparison that the wet conductors make a great deal more sparks, however, fewer but larger sparks on the dry conductors, meanwhile, conductors with $\mathrm{TiO}_{2}$ coating trigger considerably less sparks and occasionally one or two discharge sparks can be observed on the wet in particular. 

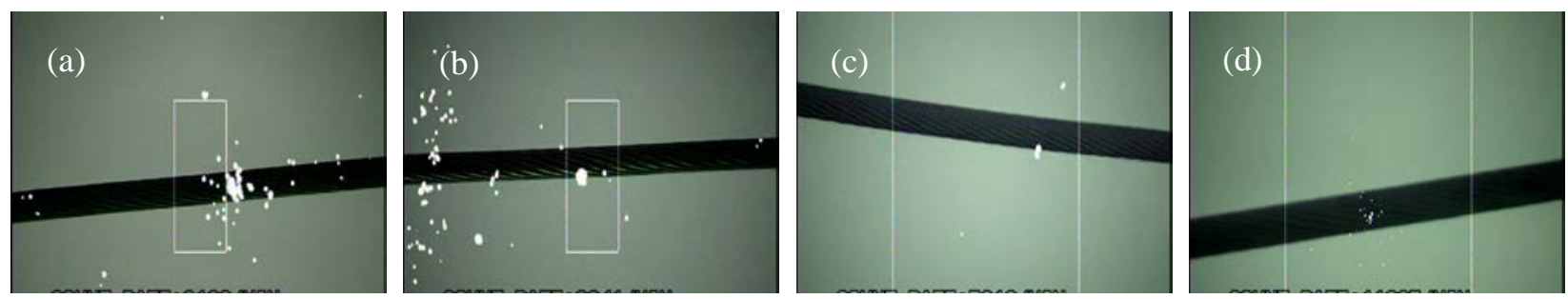

Fig. 5 UV image for corona discharge recorded under $110 \mathrm{kV}$,

(a) dry conductor and (b) wet conductor without $\mathrm{TiO}_{2}$ coating;

(b) (c) dry conductor and (d) wet conductor with $\mathrm{TiO}_{2}$ coating.

\section{Conclusions}

(1) By means of spraying $\mathrm{TiO}_{2}$ coating onto the conductor surface, the electric field force could be significantly reduced as the electric field intensity was decreased. Therefore, when it rains, the reduce in resultant of gravity and electric field force exerting downwards the droplet affixed to the conductor surface led to larger radius of curvature at the tips of water drops, resulting in less chances to get corona discharge accordingly.

(2) A coarse coating on the conductor surface was realized by spraying with $\mathrm{TiO}_{2}$, of which the roughness increased from $0.12 \mu \mathrm{m}$ to $5.89 \mu \mathrm{m}$. By this it is means that the surface wetting property was improved since the contact angle decreased from $52^{\circ}$ to $21.5^{\circ}$ accordingly, thereby, when a water drop was dropped onto this surface, it was easier to be stretched into a broad film rather than a hanging droplet, so as to reduce corona discharge.

(3) For artificial rain tests in simulating HV transmission line with voltage of $50-110 \mathrm{kV}$, it was found that conductor without coating showed discharge sparks at $50 \mathrm{kV}$ while the conductor with $\mathrm{TiO}_{2}$ coating merely sparked occasionally at $80 \mathrm{kV}$. When rainfall was made, the wet conductor made a great deal more sparks than the dry one, even so, the wet conductor with $\mathrm{TiO}_{2}$ coating made considerably less and weaker sparks than the dry conductor without coating. It is suggested that coating with $\mathrm{TiO}_{2}$ had greatly weakened the discharge intensity of the transmission line surface.

\section{Acknowledgements}

This work was supported by the Natural Science Foundation of China (51174160), Science and Technology Overall Innovation Engineering Project of Shaanxi Province (15JS080, 2014SZS09-Z02) and Open Foundation from Material Corrosion and Protection Key Laboratory of Sichuan Province (2012CL03), Excellent Doctor Degree Dissertation Research Foundation of Xi'an University of Technology; Key Laboratory Project of Education department of shaanxi province: The preparation of epoxy coatings by plasma spraying.

\section{References}

[1] Z. Y. Liu: 'Ultra-High Voltage Grid. Book', 1st edn, 253-255, 2005, Economic Press China.

[2] Miya Kiyotomi and Tana Kazuo: 'Relationship between wetting property of transmission line and audible noise’, Japan: EMCJ. , 2000, 100, 65-69.

[3] Miya Kiyotomi and Tana Kazuo: 'Reduction of audible noise by super-water-repellent treatment of transmission line surfaces’, IEEJ Trans. Power Energy, 2001, 121, 136-137.

[4] A. J. Yan, L. J. Feng, H. Y. Shen, and J. Wu: 'Surface Modification of Epoxy-Ceramic Coatings by Plasma Treatment’, Appl. Mech. Mater., 2012, 161, 167-171.

[5] S. Costil, C. Mateus, and C. Coddet: 'Ceramic/fluoropolymer composite coatings by plasma spraying’, Surf. Coat. Technol., 2006, 201, 2020-2027. 
[6] F. Vargas, H. Ageorges, P. Fournier, P. Fauchais, and M.E. Lopez: 'Mechanical and tribological performance of $\mathrm{Al}_{2} \mathrm{O}_{3}-\mathrm{TiO}_{2}$ coatings elaborated by flame and plasma spraying', Surf. Coat. Technol., 2010, 205, 1132-1136.

[7] M. Georg, G. Alexandre, and V. Robert: 'Plasma spraying of efficient photoactive $\mathrm{TiO}_{2}$ coatings', Surf. Coat. Technol., 2013, 220: 40-43.

[8] Miya. Kiyotomi and Tana. Kazuo: 'Evaluation of Audible Noise from Surface Processing Conductors for AC Overhead Transmission Line’, Electr. Eng. Japan, 2007, 159: 19-25.

[9] R. P. Feynman: 'The Feynman's Lectures on Physics', 2nd Vol., 75-77, 2005, Shanghai science and Technology Press in China.

[10]W. G. Gu, Q. Z. Ye and J. Qi, J. Li: 'Study on the Discharge Phenomena of Water Drops in Wire-plate DC Gap’, High Voltage Eng. China, 2000, 26, 22-23.

[11]L.F. Liang and P. Jiang: 'University Physics', 1st edn, 344, 2004, Fudan University Press in China.

[12]X.R. Gu, Y. Zhu, and W.L. Li: 'Surface Chemistry', 1st edn, 359-383, 2001, Science Press in China.

[13]Z. J. Guan, L. M. Wang, B. Yang, and X. D. Liang: 'Electric Field Analysis of Water Drop Corona’, IEEE T Power Deliver., 2005, 20, 964-969. 